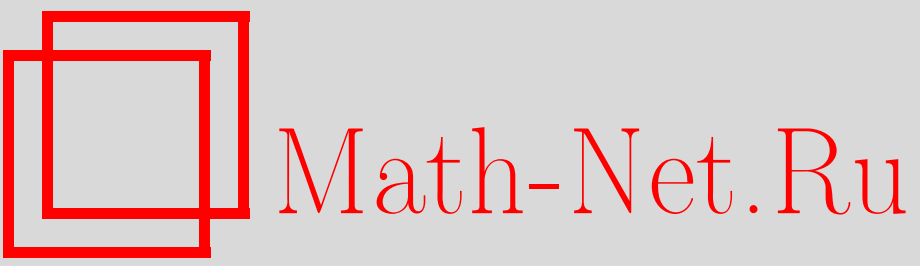

Ю. П. Чубурин, Об уравнении Шредингера для плоской пленки с предельно периодической решеткой, ТМФ, 1996, том 106, номер 1, 133-144

DOI: https://doi.org/10.4213/tmf1103

Использование Общероссийского математического портала Math-Net.Ru подразумевает, что вы прочитали и согласны с пользовательским соглашением

http://www.mathnet.ru/rus/agreement

Параметры загрузки:

IP: 54.237 .59 .107

26 апреля 2023 г., 04:59:39 


\section{ОБ УРАВНЕНИИ ШРЕДИНГЕРА ДЛЯ ПЛОСКОЙ ПЛЕНКИ С ПРЕДЕЛЬНО ПЕРИОДИЧЕСКОЙ РЕШЕТКОЙ}

Для уравнения Шредингера с потенциалом, предельно периодическим по $x_{1}, x_{2}$ и убывающим при $\left|x_{3}\right| \rightarrow \infty$, строятся решения и описываются некоторые их свойства.

\section{1. ВВЕДЕНИЕ}

Пусть $V(x), x=\left(x_{1}, x_{2}, x_{3}\right) \in \mathbb{R}^{3},-$ вешественная ограниченная функция (потенциал), периодическая по переменным $x_{j}$ с периодами $T_{j}>0, j=1,2$, и удовлетворяюшая оценке

$$
|V(x)| \leq C e^{-a\left|x_{3}\right|}, \quad x \in \mathbb{R}^{3},
$$

где $C, a>0$. Изучение оператора $H=-\Delta+V(x)$, действуюшего в $L^{2}\left(\mathbb{R}^{3}\right)$, сводится к изучению семейства операторов $H\left(k_{\|}\right)=-\Delta+V(x)$, определенных на блоховских по переменным $x_{1}, x_{2}$ функциях из $L^{2}(\Omega)$, где $\Omega=\left[0, T_{1}\right) \times\left[0, T_{2}\right) \times \mathbb{R} ;$ здесь

$$
k_{\|}=\left(k_{1}, k_{2}\right) \in\left[-\pi / T_{1}, \pi / T_{1}\right) \times\left[-\pi / T_{2}, \pi / T_{2}\right)=\Omega^{*}
$$

- (плоский) квазиимпульс. (Блоховской функцией по переменным $x_{1}, x_{2}$ называется функция, определенная на $\mathbb{R}^{3}$ и удовлетворяюшая равенству

$$
\psi\left(x_{1}+n_{1} T_{1}, x_{2}+n_{2} T_{2}, x_{3}\right)=\exp \left(i\left(k_{1} n_{1} T_{1}+k_{2} n_{2} T_{2}\right)\right) \psi(x)
$$

для всех $x \in \mathbb{R}^{3}, \quad\left(n_{1}, n_{2}\right) \in \mathbb{Z}^{2} ;$ а также сужение такой функции на $\Omega$.) См. об этом $[1,2,3]$.

В дальнейшем под $H, H\left(k_{\|}\right)$будет также пониматься соответствуюшее дифференциальное выражение. Через $\sigma(A)$ и $\sigma_{\text {ess }}(A)$ будем обозначать соответственно спектр и существенный спектр оператора $A$. Заметим, что $\sigma_{\text {ess }}\left(H\left(k_{\|}\right)\right)=\left[k_{\|}^{2}, \infty\right)[2]$ и

$$
\sigma(H)=\bigcup_{k_{\|} \in \Omega^{*}} \sigma\left(H\left(k_{\|}\right)\right)
$$

(см. $[3,2])$. Следовательно, для любого $E \in \sigma(H) \cap(-\infty, 0)$ сушествует блоховское по $x_{1}, x_{2}$ решение уравнения Шредингера

$$
H \psi=E \psi
$$


сужение которого на $\Omega$ принадлежит $L^{2}(\Omega)$ (и даже экспоненциально убывает при $\left|x_{3}\right| \rightarrow \infty$, см. ниже теорему 3$)$. Далее, если $E>0$, то для некоторого $k_{\|} \in \Omega^{*}$ имеем $E>k_{\|}^{2}$. Согласно $[4,5]$ для всех таких $E$ за исключением, возможно, не более чем счетного множества точек $\mathcal{N}\left(k_{\|}\right) \subset\left(k_{\|}^{2}, \infty\right)$, не имеющего предельных точек в промежутке $\left[k_{\|}^{2}, \infty\right)$, существует ограниченное блоховское по $x_{1}, x_{2}$ решение уравнения $(2)$, не стремящееся к нулю при $x_{3} \rightarrow \pm \infty$ (последнее в силу того, что асимптотика решений представляет собой конечную сумму плоских волн). Итак, в силу вышесказанного, а также доказанной ниже леммы 5 , для любого $E \in \sigma(H) \backslash\{0\}$ сушествует блоховское по $x_{1}, x_{2}$ решение уравнения (2), экспоненциально убьваюшее при $\left|x_{3}\right| \rightarrow \infty$, если $E<0$, и ограниченное, если $E>0$.

В настоящей работе речь пойдет о решениях уравнения (2) в случае предельно периодического по переменным $x_{1}, x_{2}$ и убываюшего при $\left|x_{3}\right| \rightarrow \infty$ потенциала $V(x)$ (случай предельной периодичности по всем переменным см. в [6]). Заметим, что гамильтониан $H$ можно рассматривать как оператор энергии электрона в плоской пленке с нарушенной (например, примесями) кристаллической структурой.

Будем говорить, что функция $V(x)$, определенная на $\mathbb{R}^{3}$, является предельно периодической по переменным $x_{1}, x_{2}$, если существует последовательность периодических по $x_{1}, x_{2}$ непрерывных ограниченных функций $V_{N}(x), N=1,2, \ldots$, равномерно сходящаяся к $V(x)$. Подобно тому, как и в одномерном случае [7], можно доказать, что $V(x)$ является предельно периодической в том и только в том случае, если существует последовательность непрерывных ограниченных функций $W_{N}(x), N=1,2, \ldots$, периодических с периодами $T_{j}^{(N)}>0$ по переменным $x_{j}, j=1,2$, таких, что

$$
V(x)=\sum_{N=1}^{\infty} W_{N}(x)
$$

причем ряд

$$
\sum_{N=1}^{\infty}\left\|W_{N}\right\|_{L^{\infty}\left(\mathbb{R}^{3}\right)}
$$

сходится и для всех $N$ выполнено $T_{j}^{(N+1)} / T_{j}^{(N)} \in \mathbb{Z}, j=1,2$. При этом, если функции $V_{N}(x)$ удовлетворяют оценкам вида $(1)$, то и $W_{N}(x)$ удовлетворяют оценкам того же вида. Заметим, что в данном случае $\lim _{\left|x_{3}\right| \rightarrow \infty} V(x)=0$.

Наконец, заметим, что если $V(x)$ - предельно периодический потенциал по $x_{1}, x_{2}$,

$$
H=-\Delta+V(x), \quad H_{N}=-\Delta+\sum_{n=1}^{N} W_{n}(x),
$$

то $\sigma(H)$ состоит из пределов всевозможных сходяшихся последовательностей чисел $\lambda_{N} \in \sigma\left(H_{N}\right), N=1,2, \ldots$, и только из них. Это легко следует из наличия сильной резольвентной сходимости $H_{N}$ к $H($ см $[8])$. В дальнейшем полагаем

$$
V_{N}(x)=\sum_{n=1}^{N} W_{n}(x)
$$


Введем в случае $T_{j}=T_{j}^{(N)}, j=1,2$, обозначения $\Omega_{N}=\Omega, \Omega_{N}^{*}=\Omega^{*}$. Оператор $H_{N}\left(k_{\|}\right)=-\Delta+V_{N}(x)$, где $k_{\|} \in \Omega_{N}^{*}$, действуюший в $L^{2}\left(\Omega_{N}\right)$, можно также рассматривать в пространстве $L^{2}\left(\Omega_{N+M}\right), M>0$. Соответственно вместо $k_{\|}$следует брать

$$
\tilde{k}_{\|}=k_{\|} \bmod \left(2 \pi / T_{N+M}^{(1)}, 2 \pi / T_{N+M}^{(2)}\right) .
$$

Вектор $\tilde{k}_{\|}$будем называть образом $k_{\|}$в $\Omega_{N+M}^{*}$.

Заметим, что сформулированные ниже теоремы 1, 2 аналогичны теоремам 1, 2 работы [6]. Приведем другие в какой-то мере близкие утверждения: для теоремы 1 это результат статьи [9], в которой доказано сушествование решения уравнения Шредингера с квазипериодическим потенциалом в минимальной точке спектра; а для теоремы 2 результат [10] о сушествовании для широкого класса потенциалов полиномиально ограниченных решений уравнения Шредингера для плотного подмножества точек спектра.

\section{2. ФОРМУЛИРОВКА РЕЗУЛЬТАТОВ}

Предположим, что заданы периоды $T_{j}^{(N)}>0$ такие, что $T_{j}^{(N+1)} / T_{j}^{(N)} \in \mathbb{Z}$ для всех $N$ и $j=1,2$. Пусть, далее, $K_{N} \subset L^{\infty}\left(\Omega_{N}\right)$ - произвольные компактные множества, состоящие из непрерывных функций, для которых вьполнены оценки вида (1) (с различными $C, \alpha) ; N=1,2, \ldots$.

Теорема 1. Предположим, что периодическая по $x_{j}$ с периодами $T_{j}^{(1)}, j=1,2$, функиия $U(x)$ удовлетворяет оценке вида $(1)$, а также неравенству

$$
\alpha=\int_{\Omega_{1}} U(x) d x<0 .
$$

Существуют числа $\varepsilon_{N}>0, \quad N=1,2, \ldots$, такие, что если $0<\varepsilon \leq \varepsilon_{1}$, $W_{1}(x)=\varepsilon U(x), \quad\left\|W_{N}(x)\right\|_{L^{\infty}(\Omega)} \leq \varepsilon_{N} u W_{N}(x) \in K_{N}, \quad N=2,3, \ldots$, а потенииал $V(x)$ имеет вид (3), то для $E=\min \sigma(H)$ существует ненулевое решение $\psi(x)$ уравнения ШШредингера $(2)$ в $\mathbb{R}^{3}$ такое, что $|\psi(x)|$ - предельно периодическая функиия по переменным $x_{1}, x_{2}$.

Tеорема 2. Существуют такие числа $\varepsilon_{N}>0, N=1,2, \ldots$, что если $\left\|W_{N}(x)\right\|_{L^{\infty}(\Omega)} \leq \varepsilon_{N} u W_{N}(x) \in K_{N}, \quad N=1,2, \ldots$, то найдется плотное в множестве $\sigma(H) \cap(-\infty, 0)$ множество $X \subset \sigma(H)$ такое, что для любого $E \in X$ имеется ненулевое решение $\psi(x)$ уравнения (2), причем $|\psi(x)|-$ предельно периодическая функиия по $x_{1}, x_{2}$.

Теорема 3. Пусть $V(x)$ - потенциал, удовлетворяющий неравенству (1), $\psi(x)$ - ограниченное решение уравнения ШІредингера (2) $c E<0$. Тогда справедлива оченка

$$
|\psi(x)| \leq C_{1} e^{-a_{1}\left|x_{3}\right|}, \quad x \in \mathbb{R}^{3},
$$

әде $C_{1}, a_{1}>0$.

СледСтвиЕ. Пусть $V(x)$ - предельно периодический потенциал, удовлетворяющий оценке (1). Тогда все предельно периодические по модулю по переменным $x_{1}, x_{2}$ решения уравнения ШШредингера (2) с $E<0$ удовлетворяют оценке вида (4).

Положим $\sqrt{V(x)}=\sqrt{|V(x)|} \operatorname{sgn} V(x)$. 
ТеОрема 4. Пусть в выражсении (3) функиия $W_{1}(x)$ удовлетворяет оценке вида (1) (а в остальном произвольна), а функиии $W_{N+1}(x), \quad N \geq 1$, въбираются удовлетворяющими оценке этого жее вида (с различными $C$, a) и так, чтобы числа $\varepsilon_{N+1}=\varepsilon_{N+1}\left(V_{N}\right)$ в неравенствах

$$
\begin{gathered}
\left\|\sqrt{\left|V_{N+1}(x)\right|}-\sqrt{\left|V_{N}(x)\right|}\right\|_{L^{\infty}\left(\Omega_{N+1}\right)} \leq \varepsilon_{N+1}, \\
\left\|\sqrt{V_{N+1}(x)}-\sqrt{V_{N}(x)}\right\|_{L^{2}\left(\Omega_{N+1}\right)} \leq \varepsilon_{N+1}
\end{gathered}
$$

бълли достаточно маль. Тогда уравнение IIредингера (2) для почти всех $E>0$ имеет не стремящееся к нулю при фиксированных $x_{1}, x_{2}$ и при $x_{3} \rightarrow \pm \infty$ решение такое, что $|\psi(x)|$ - предельно периодическая функиия по $x_{1}, x_{2}$.

\section{3. ДОКАЗАТЕЛЬСТВА ТЕОРЕМ}

Согласно [11] оператор $H_{1}\left(k_{\|}\right)=-\Delta+W_{1}(x)$ имеет для $k_{\|}$вне произвольно малой окрестности гранишы множества $\Omega_{1}^{*}$ и малых $\varepsilon>0$ единственное собственное значение $E_{\varepsilon}\left(k_{\|}\right)<k_{\|}^{2}$ кратности единица, аналитически зависяшее от $k_{\|}, \varepsilon$ и имеющее вид

$$
E_{\varepsilon}\left(k_{\|}\right)=k_{\|}^{2}-\frac{\varepsilon^{2} \alpha^{2}}{4}+f\left(k_{\|}, \varepsilon\right)
$$

где функция $f\left(k_{\|}, \varepsilon\right)$ удовлетворяет оценке

$$
\left|f\left(k_{\|}, \varepsilon\right)\right| \leq C \varepsilon^{3}
$$

причем $C$ не зависит от $k_{\|}$в некоторой комплексной (в $\mathbb{C}^{2}$ ) окрестности нуля. Вследствие интегральной формулы Коши неравенства, аналогичные (6), справедливы в некоторой окрестности нуля для производных $\partial^{M_{1}+M_{2}} f\left(k_{\|}, \varepsilon\right) / \partial k_{1}^{M_{1}} \partial k_{2}^{M_{2}}$ любых порядков.

В силу $(5),(6)$ очевидно, что функция $E_{\varepsilon}\left(k_{\|}\right)$для малых $\varepsilon$ имеет хотя бы один отрицательный минимум.

Лемма 1. Минимум функции $E_{\varepsilon}\left(k_{\|}\right)$для малых в единствен.

ДокАЗАТЕЛЬСТво. В силу (5) и оценок вида (6) для производных функции $f\left(k_{\|}, \varepsilon\right)$ по $k_{1}, k_{2}$ отображение $(-1 / 2) \operatorname{grad}_{\|} f\left(k_{\|}, \varepsilon\right)$, где $\operatorname{grad}_{\|}=\left(\partial / \partial k_{1}, \partial / \partial k_{2}\right)$, для достаточно малых $\varepsilon$ переводит множество

$$
\mathcal{F}_{\varepsilon}=\left\{k_{\|} \in \Omega_{1}^{*}: E_{\varepsilon}\left(k_{\|}\right) \leq-\sigma\right\}
$$

в себя и является сжимающим, где $\sigma>0$ выбрано настолько малым, что $\mathcal{F}_{\varepsilon}$ содержит все точки (глобального) минимума функции $E_{\varepsilon}\left(k_{\|}\right)$, а также некоторую окрестность точки $k_{\|}=0$. Вследствие принципа сжимающих отображений сушествует единственная стационарная точка функции $E_{\varepsilon}\left(k_{\|}\right)$в $\mathcal{F}_{\varepsilon}$, поскольку такие точки согласно (5) определяются уравнением $k_{\|}=(-1 / 2) \operatorname{grad}_{\|} f\left(k_{\|}, \varepsilon\right)$. Лемма доказана. 
Заметим, что из упомянутых вьше оценок для производных функции $f\left(k_{\|}, \varepsilon\right)$ по $k_{1}$, $k_{2}$, а также из (5) вытекает равенство

$$
\operatorname{det}\left(\frac{\partial^{2} E_{\varepsilon}\left(k_{\|}\right)}{\partial k_{i} \partial k_{j}}\right)_{i, j=1}^{2}=4+O\left(\varepsilon^{3}\right)
$$

причем в неравенстве $\left|O\left(\varepsilon^{3}\right)\right| \leq C \varepsilon^{3}$ константу $C$ можно считать не зависяшей от $k_{\|} \in \mathcal{F}_{\varepsilon}$.

Положим $R_{N}\left(k_{\|}, E\right)=\left(H_{N}\left(k_{\|}\right)-E\right)^{-1}$. Пусть $K$ - компактное множество в $\mathbb{C} \backslash \sigma\left(H_{N}\left(k_{\|}\right)\right)$.

Лемма 2. Для любого $\varepsilon>0$ существует $\delta>0$ такое, что из

$$
\left|k_{\|}^{\prime}-k_{\|}\right| \leq \delta, \quad\left\|W_{N+1}(x)\right\|_{L^{\infty}\left(\Omega_{N+1}\right)} \leq \delta
$$

вытекают существование резольвенты $R_{N+1}\left(k_{\|}^{\prime}, E\right)$ и неравенство

$$
\left\|R_{N+1}\left(k_{\|}^{\prime}, E\right)-R_{N}\left(k_{\|}, E\right)\right\| \leq \varepsilon
$$

сразу для всех $E \in K$ (в неравенстве $(7)$ оператор $R_{N}\left(k_{\|}, E\right)$ рассматривается в $\left.L^{2}\left(\Omega_{N+1}\right)\right)$.

ДоКАЗАТЕЛЬСТво. С помощью хорошо известного унитарного преобразования [3] перейдем к операторам

$$
\widetilde{H}_{N}\left(k_{\|}\right)=e^{-i\left(k_{\|}, x_{\|}\right)} H_{N}\left(k_{\|}\right) e^{i\left(k_{\|}, x_{\|}\right)}=-\Delta-2 i\left(k_{\|}, \operatorname{grad}_{\|}\right)+k_{\|}^{2}+V_{N}(x),
$$

где $x_{\|}=\left(x_{1}, x_{2}\right), \quad \operatorname{grad}_{\|}=\left(\partial / \partial x_{1}, \partial / \partial x_{2}\right)$. Резольвенту $\widetilde{H}_{N}\left(k_{\|}\right)$обозначим через $\widetilde{R}_{N}\left(k_{\|}, E\right)$. В силу резольвентного тождества имеем

$$
\begin{aligned}
& \widetilde{R}_{N+1}\left(k_{\|}^{\prime}, E\right)-\widetilde{R}_{N}\left(k_{\|}, E\right)=-\widetilde{R}_{N+1}\left(k_{\|}^{\prime}, E\right) \times \\
& \quad \times\left(-2 i\left(k_{\|}^{\prime}-k_{\|}, \operatorname{grad}_{\|}\right)+k_{\|}^{\prime 2}-k_{\|}^{2}+W_{N+1}(x)\right) \widetilde{R}_{N}\left(k_{\|}, E\right) .
\end{aligned}
$$

Докажем равномерную по $E \in K$ ограниченность операторов $\operatorname{grad}_{\|} \widetilde{R}_{N}\left(k_{\|}, E\right)$ или эквивалентно операторов $\operatorname{grad}_{\|} R_{N}\left(k_{\|}, E\right)$; данное утверждение вместе с равенством (8) докажет лемму. Вследствие равенства

$$
R_{N}\left(k_{\|}, E\right)=R_{0}\left(k_{\|}, E\right)-R_{0}\left(k_{\|}, E\right) V_{N}(x) R_{N}\left(k_{\|}, E\right),
$$

где $R_{0}\left(k_{\|}, E\right)$ - резольвента оператора $H_{0}\left(k_{\|}\right)=-\Delta$, достаточно доказать равномерную ограниченность операторов $\operatorname{grad}_{\|} R_{0}\left(k_{\|}, E\right)$. Имеем

$$
R_{0}\left(k_{\|}, E\right) \psi(x)=\sum_{n_{\|} \in \mathbb{Z}^{2}} \exp \left(i\left(k_{\|}+2 \pi n_{\|}, x_{\|}\right)\right) \frac{1}{\sqrt{2 \pi}} \int_{-\infty}^{\infty} \frac{e^{-i p_{3} x_{3}} \widehat{\psi}_{n_{\|}}\left(p_{3}\right) d p_{3}}{-E+\left(k_{\|}+2 \pi n_{\|}\right)^{2}+p_{3}^{2}},
$$

где

$$
\widehat{\psi}_{n_{\|}}\left(p_{3}\right)=\frac{1}{\sqrt{2 \pi}} \int_{-\infty}^{\infty}\left(\int_{(0,1)^{2}} \exp \left(-i\left(k_{\|}+2 \pi n_{\|}, x_{\|}\right)\right) \psi(x) d x_{\|}\right) e^{i p_{3} x_{3}} d x_{3} .
$$

Требуемая оценка легко получается после дифференцирования ряда (9) с использованием равенства Парсеваля и изометричности преобразования Фурье (ср. ниже доказательство леммы 5). 
Лемма 3. Пусть $\psi_{N}(x) \in L^{2}\left(\Omega_{N+1}\right)$ - собственная функиия единичной нормы оператора $H_{N}\left(k_{\|}\right)$(рассматриваемого в $L^{2}\left(\Omega_{N+1}\right)$ ), отвечающая собственному значению $E_{N}\left(k_{\|}\right)<k_{\|}^{2}$ кратности единица. Для любого $\varepsilon>0$ существует $\delta>0$ такое, что если

$$
\left|k_{\|}^{\prime}-k_{\|}\right| \leq \delta, \quad\left\|W_{N+1}(x)\right\|_{L^{\infty}\left(\Omega_{N+1}\right)} \leq \delta
$$

то у оператора $H_{N+1}\left(k_{\|}^{\prime}\right)$ существует собственная функция $\psi_{N+1}(x) \in L^{2}\left(\Omega_{N+1}\right)$ единичной нормы, отвечающая собственному значению $E_{N+1}\left(k_{\|}^{\prime}\right)$ кратности единича, причем справедливы неравенства

$$
\begin{aligned}
\left|E_{N+1}\left(k_{\|}^{\prime}\right)-E_{N}\left(k_{\|}\right)\right| & \leq \varepsilon \\
\left\|\psi_{N+1}(x)-\psi_{N}(x)\right\|_{L^{2}\left(\Omega_{N+1}\right)} & \leq \varepsilon .
\end{aligned}
$$

ДокАЗАТЕЛЬСТво вытекает из леммы 2 и стандартных рассуждений теории возмущений, использующих формулу Рисса - см., например, [3].

Лемма 4. В условиях леммы 3 имеет место также неравенство

$$
\left\|\psi_{N+1}(x)-\psi_{N}(x)\right\|_{L^{\infty}\left(\Omega_{N+1}\right)} \leq \varepsilon
$$

ДокАЗАТЕльство. Обозначим через $\Gamma_{N+1}\left(x-y, k_{\|}, E\right)$ ядро резольвенты $R_{0}\left(k_{\|}, E\right)$ оператора $H_{0}\left(k_{\|}\right)$, рассматриваемого в $L^{2}\left(\Omega_{N+1}\right)$. Заметим, что при фиксированном $y$ ядро аналитически зависит от $k_{\|}, E$ как $L^{2}\left(\Omega_{N+1}\right)$-значная функция (см. [4]). Имеем

$$
\begin{aligned}
\psi_{N+1}(x) & =\int_{\Omega_{N+1}} \Gamma_{N+1}\left(x-y, k_{\|}^{\prime}, E_{N+1}\left(k_{\|}^{\prime}\right)\right) V_{N+1}(y) \psi_{N+1}(y) d y \\
\psi_{N}(x) & =\int_{\Omega_{N+1}} \Gamma_{N+1}\left(x-y, k_{\|}, E_{N}\left(k_{\|}\right)\right) V_{N}(y) \psi_{N}(y) d y
\end{aligned}
$$

Нужное неравенство легко получается из приведенных соотношений с помощью леммы 3 и неравенства Коши-Буняковского.

ДОКАЗАТЕЛЬСТВА ТЕОРЕМ 1, 2 вПолне аналогичны доказательствам соответствующих теорем в [6] с использованием сформулированных выше в данном разделе утверждений (лемма 1 относится лиш к теореме 1). В теореме 2 также надо использовать факт конечности числа собственных значений операторов $H_{N}\left(k_{\|}\right)$, меньших $k_{\|}^{2}[5]$, и то обстоятельство, что они неявно задаются нулями аналитической функции аргументов $k_{\|}, E[4]$. 
ДОКАЗАТЕЛЬСТВО ТЕОРЕМЫ 3. ОбоЗначим через

$$
G(x-y, E)=\exp (-\sqrt{|E|}|x-y|) / 4 \pi|x-y|
$$

ядро резольвенты оператора $-\Delta$ в $L^{2}\left(\mathbb{R}^{3}\right)$. Пусть для простоты $T_{1}=T_{2}=1$. Имеем

$$
\psi(x)=-\int_{\mathbb{R}^{3}} G(x-y, E) V(y) \psi(y) d y,
$$

откуда

$$
\begin{aligned}
|\psi(x)| & \leq C \int_{\mathbb{R}^{3}} G(x-y, E) e^{-a\left|y_{3}\right|} d y= \\
& =C \int_{\Omega}\left(\sum_{n_{\|} \in \mathbb{Z}^{2}} G\left(x-y-\left(n_{\|}, 0\right), E\right)\right) e^{-a\left|y_{3}\right|} d y= \\
& =C \int_{\Omega} \Gamma(x-y, 0, E) e^{-a\left|y_{3}\right|} d y,
\end{aligned}
$$

где $\Omega=[0,1) \times \mathbb{R}, \Gamma(x-y, 0, E)-$ ядро резольвенты оператора $H_{0}\left(k_{\|}\right)=-\Delta$ в $L^{2}(\Omega)$ для $k_{\|}=0$ (см. [4]). Нужная оценка вытекает после применения неравенства Коши-Буняковского из результатов [2] (оценка доказывается с помошью равенства Парсеваля и замены ряда по $n_{\|}$на интеграл - см. также [4]). Теорема доказана.

Обозначим через $\Gamma\left(x-y, k_{\|}, E+i 0\right)$ естественное продолжение ядра резольвенты оператора $H_{0}\left(k_{\|}\right)$, действуюшего в $L^{2}(\Omega)$, в точку спектра

$$
E \in\left(k_{\|}^{2}, \infty\right) \backslash\left\{\left(k_{\|}+2 \pi\left(n_{1} / T_{1}, n_{2} / T_{2}\right)\right)^{2}: n_{\|} \in \mathbb{Z}^{2}\right\}
$$

(в точках $E=\left(k_{\|}+2 \pi\left(n_{1} / T_{1}, n_{2} / T_{2}\right)\right)^{2}$ функция $\Gamma\left(x-y, k_{\|}, E+i 0\right)$ не определена). О возможности такого продолжения см. [4], а также приведенные ниже рассуждения. Пусть потенциал $V(x)$ удовлетворяет оценке (1). Рассмотрим модифицированное уравнение Липпмана-Швингера [12] для блоховских функций

$$
\varphi(x)=\varphi_{0}(x)-\int_{\Omega_{N}} \sqrt{V(x)} \Gamma_{N}\left(x-y, k_{\|}, E+i 0\right) \sqrt{V(y)} \varphi(y) d y,
$$

где $\varphi_{0}(x)=\sqrt{|V(x)|} \exp \left(i\left(\left(k_{\|}, \sqrt{E-k_{\|}^{2}}\right), x\right)\right) ;$ решение $\varphi(x)$ ишется в классе $L^{2}\left(\Omega_{N}\right)$ (соответствуюшее решение $\psi(x)$ уравнения Липпмана-Швингера связано с $\varphi(x)$ равенством $\varphi(x)=\sqrt{|V(x)|} \psi(x)$ ). Под множеством $\mathcal{N}\left(k_{\|}\right)$, о котором шла речь во введении, следует понимать объединение множества точек $E>k_{\|}^{2}$, для которых уравнение (10) с $\varphi_{0}(x)=0$ имеет ненулевое решение (при этом множество пар $\left(k_{\|}, E\right)$ для таких $E$ локально есть множество нулей аналитической функции - см. [4] - и, следовательно, замкнуто) с множеством точек $E=\left(k_{\|}+2 \pi\left(n_{1} / T_{1}, n_{2} / T_{2}\right)\right)^{2}$, где $n_{\|}=\left(n_{1}, n_{2}\right) \in \mathbb{Z}^{2}$. Заметим, что точки $E$, в которых имеется ненулевое решение уравнения (10) с $\varphi_{0}(x)=0$, являются собственными значениями (с помошью формулы Грина можно доказать, что коэффициенты при плоских волнах, дающих асимптотику соответствующих решений 
уравнения Шредингера - см. [4] - обрашаются в нуль, и, таким образом, решение принадлежит $\left.L^{2}\left(\Omega_{N}\right)\right)$. Примеры таких собственных значений легко построить, беря потенциал, зависящий только от $x_{3}$.

Обозначим через $\mathcal{M}\left(k_{\|}\right)$объединение $\mathcal{N}\left(k_{\|}\right)$и собственных значений дискретного спектра $E_{\nu}\left(k_{\|}\right)<k_{\|}^{2}, \quad \nu=1,2, \ldots$ Согласно [4] и теореме Фредгольма для $E \in$ $\left(k_{\|}^{2}, \infty\right) \backslash \mathcal{M}\left(k_{\|}\right)$уравнение $(10)$ имеет единственное решение.

При доказательстве следуюшего утверждения для простоты записи полагаем $T_{1}^{(N)}=$ $T_{2}^{(N)}=1, \Gamma_{N}\left(x, k_{\|}, E\right)=\Gamma\left(x, k_{\|}, E\right)$.

ЛЕмма 5. (ср. периодический случай [3]). Множсество $\Omega^{*} \times \mathcal{M}\left(k_{\|}\right)$не содержит подмножеств вида $\left\{\left(k_{1}^{(n)}, k_{2}, E\right)\right\}_{n=1}^{\infty}$, әде $\left\{k_{1}^{(n)}\right\} \subset[-\pi, \pi)-$ сходящаяся последовательность с пределом $k_{1}^{*} \neq 0$ (в частности, у собственных значений нет участков постоянства по $\left.k_{1}\right)$.

ДокАЗАТЕЛЬСТво. Без ограничения общности можно считать, что $E \neq\left(k_{\|}+\right.$ $\left.2 \pi n_{\|}\right)^{2}, n_{\|} \in \mathbb{Z}^{2}$. Для малых по модулю $s, \varepsilon$ сушествует аналитическое продолжение по переменным $k_{1}+i s, E+i \varepsilon L^{2}(\Omega \times \Omega)$-значной функции

$$
\sqrt{|V(x)|} \Gamma\left(x-y, k_{\|}, E+i 0\right) \sqrt{V(y)} \text {; }
$$

это легко усмотреть из вида ядер резольвент, приведенных (в более обшем случае) в [13]

Рассмотрим область в $\mathbb{C}$ вида $\omega=(\alpha, \beta)+i(-\delta, \infty)$, где $(\alpha, \beta)$ содержит $k_{1}^{*}$, причем $[\alpha, \beta] \cap\{0\}=\varnothing$, а $\delta>0$ достаточно мало. Положим

$$
\widetilde{H}\left(k_{\|}\right)=e^{-i\left(k_{\|}, x_{\|}\right)} H\left(k_{\|}\right) e^{i\left(k_{\|}, x_{\|}\right)}
$$

(см. доказательство леммы 2) и обозначим через $\widetilde{R}_{0}\left(k_{\|}, E\right)$ резольвенту оператора $\widetilde{H}_{0}\left(k_{\|}\right)$. Для доказательства леммы достаточно доказать, что для операторнозначной функции

$$
\sqrt{|V(x)|} \widetilde{R}_{0}\left(k_{\|}, E+i 0\right) \sqrt{V(x)}
$$

с ядром

$$
e^{-i\left(k_{\|}, x_{\|}\right)} \sqrt{|V(x)|} \Gamma\left(x-y, k_{\|}, E+i 0\right) \sqrt{V(y)} e^{i\left(k_{\|}, y_{\|}\right)}
$$

имеется аналитическое продолжение по $k_{1}+i s$ в область $\omega$, причем вьполнено условие

$$
\left\|\sqrt{|V(x)|} \widetilde{R}_{0}\left(k_{1}+i(s, 0), E+i 0\right) \sqrt{V(x)}\right\| \rightarrow 0, \quad s \rightarrow \infty .
$$

Действительно, в этом случае совокупность точек $k_{1}+i s \in \omega$, для которых при фиксированных $k_{2}, E$

$$
\begin{aligned}
& \operatorname{ker}\left\{1+\sqrt{|V(x)|} \widetilde{R}_{0}\left(k_{\|}+i(s, 0), E+i 0\right) \sqrt{V(x)}\right\}= \\
& \quad=\operatorname{ker}\left\{1+\sqrt{|V(x)|} R_{0}\left(k_{\|}+i(s, 0), E+i 0\right) \sqrt{V(x)}\right\} \neq\{0\},
\end{aligned}
$$

не совпадает с $\omega$ и в силу аналитической теоремы Фредгольма [8] не имеет предельных точек, что и докажет лемму. 
Осталось доказать (13); из полученных при этом оценок и теоремы Вейерштрасса (в векторном варианте) об аналитичности ряда, составленного из аналитических функций, будет следовать возможность аналитического продолжения операторнозначной функции (11) для произвольных $s>0$. Пусть $s>\left|\varepsilon / k_{1}\right|>0, \varphi(x) \in L^{2}(\Omega)$ и $\psi(x)=\sqrt{V(x)} e^{i\left(k_{\|}, x_{\|}\right)} \varphi(x)$. Оценим, пользуясь (9),

$$
\begin{aligned}
& \left\|\sqrt{|V(x)|} \widetilde{R}_{0}\left(k_{\|}+i(s, 0), E+i \varepsilon\right) \sqrt{V(x)} \varphi(x)\right\|_{L^{2}(\Omega)}= \\
& =\| \sqrt{|V(x)|} \sum_{n_{\|} \in \mathbb{Z}^{2}} \exp \left(i\left(2 \pi n_{\|}, x_{\|}\right)\right) \times \\
& \quad \times \frac{1}{\sqrt{2 \pi}} \int_{-\infty}^{\infty} \frac{e^{-i p_{3} x_{3}} \widehat{\psi}_{n_{\|}}\left(p_{3}\right) d p_{3}}{-E-i \varepsilon+\left(k_{\|}+2 \pi n_{\|}\right)^{2}+p_{3}^{2}-s^{2}+2 i s\left(k_{1}+2 \pi n_{1}\right)} \|_{L^{2}(\Omega)}^{2} \leq \\
& \leq \frac{C_{1}}{\left(s-\left|\varepsilon / 2 k_{1}\right|\right)^{2}} \sum_{n_{\|} \in \mathbb{Z}^{2}}\left\|\widehat{\psi}_{n_{\|}}\left(p_{3}\right)\right\|_{L^{2}(\mathbb{R})}^{2} \leq \frac{4 C_{1}}{|s|^{2}}\|\psi\|_{L^{2}(\Omega)}^{2}
\end{aligned}
$$

равномерно по всем достаточно малым $\varepsilon$, что позволяет перейти в полученном неравенстве к пределу при $\varepsilon \rightarrow 0$, используя при этом ядро вида $(12)-$ см. начало доказательства. Отсюда сразу следует (13). Лемма доказана.

Лемма 6. Пусть $A \subset \mathbb{R}$ - ограниченное множество такое, что для некоторого $\sigma_{0}>0$ и всех $E \in A$ и $n_{\|} \in \mathbb{Z}^{2}$ выполнено неравенство

$$
\left|E-\left(k_{\|}+2 \pi\left(n_{1} / T_{1}^{N+1}, n_{2} / T_{2}^{N+1}\right)\right)^{2}\right| \geq \sigma_{0} .
$$

Пусть, далее, потенциал $V_{N}(x)$ фиксирован. Для любого $\varepsilon>0$ существует $\delta>0$ такое, что если

$$
\begin{array}{r}
\left\|\sqrt{V_{N+1}(x)}\right\|_{L^{\infty}\left(\Omega_{N+1}\right)}\left\|\sqrt{V_{N+1}(x)}-\sqrt{V_{N}(x)}\right\|_{L^{2}\left(\Omega_{N+1}\right)} \leq \delta, \\
\left\|\sqrt{\left|V_{N+1}(x)\right|}-\sqrt{\left|V_{N}(x)\right|}\right\|_{L^{2}\left(\Omega_{N+1}\right)} \leq \delta,
\end{array}
$$

mo

$$
\begin{aligned}
& \| \sqrt{\left|V_{N+1}(x)\right|} \Gamma_{N+1}\left(x-y, k_{\|}, E+i 0\right) \sqrt{V_{N+1}(y)}- \\
& \quad-\sqrt{\left|V_{N}(x)\right|} \Gamma_{N+1}\left(x-y, k_{\|}, E+i 0\right) \sqrt{V_{N}(y)} \|_{L^{2}\left(\Omega_{N+1} \times \Omega_{N+1}\right)} \leq \varepsilon
\end{aligned}
$$

равномерно по $E \in A$. 
ДокАЗАТЕЛЬСТво. Имеем

$$
\Gamma_{N+1}\left(x, k_{\|}, E+i 0\right)=\Gamma_{N+1}^{(1)}\left(x, k_{\|}, E+i 0\right)+\Gamma_{N+1}^{(2)}\left(x, k_{\|}, E\right)
$$

причем нормы

$$
\left\|\Gamma_{N+1}^{(1)}\left(x, k_{\|}, E+i 0\right)\right\|_{L^{\infty}\left(\Omega_{N+1}\right)}, \quad\left\|\Gamma_{N+1}^{(2)}\left(x, k_{\|}, E\right)\right\|_{L^{2}\left(\Omega_{N+1}\right)}
$$

ограничены равномерно по $E \in A$ (см. [4]). Требуемое неравенство получается с помощью простых оценок, используюших приведенное утверждение.

ДокаЗАТЕЛЬСТво теОремы 4 . Через $\mathcal{M}_{N}\left(k_{\|}\right)$будем обозначать множество $\mathcal{M}\left(k_{\|}\right)$ для оператора $H_{N}\left(k_{\|}\right)$. Для простоты обозначений образы $\tilde{k}_{\|} \in \Omega_{N+1}^{*}, \tilde{\tilde{k}}_{\|} \in \Omega_{N+2}^{*}$ и т.д. точки $k_{\|} \in \Omega_{N}^{*}$ будем обозначать той же буквой. Выберем точку $k_{\|}^{(1)}=$ $\left(k_{1}^{(1)}, k_{2}^{(1)}\right) \in \Omega_{1}^{*}$ так, чтобы у нее, а также у всех ее образов в $\Omega_{N}^{*}, N \geq 2$, выполнялось условие $k_{1}^{(1)} \neq 0$; выберем также отрезок $\Delta_{1}^{(1)}=\left[a_{1}, b_{1}\right] \subset\left(\left(k_{\|}^{(1)}\right)^{2}, \infty\right)$. Пусть $\Delta_{1}^{(1)} \cap \mathcal{M}_{1}\left(k_{\|}^{(1)}\right)=\left\{E_{i}^{(1)}\right\}_{i=1}^{n_{1}}$. С помошью леммы 5 легко доказать от противного, что сушествует точка $k_{\|}^{(2)} \in \Omega_{1}^{*}$, сколь угодно близкая к $k_{\|}^{(1)}$, в частности, такая, что $\left(k_{\|}^{(2)}\right)^{2}<a_{1}$, для которой выполнено условие $\mathcal{M}_{1}\left(k_{\|}^{(2)}\right) \cap\left\{E_{i}^{(1)}\right\}_{i=1}^{n_{1}}=\varnothing$; при этом можно считать, что для нее и всех ее образов $k_{1}^{(2)} \neq 0$. Выберем (достаточно малое) $\varepsilon>0$, а также множество $\Delta_{1}^{(2)}$, полученное из $\Delta_{1}^{(1)}$ выбрасьванием открытых интервалов, содержащих точки $E_{i}^{(1)}, i=1, \ldots, n_{1}$, таких, что при этом $\mu\left(\Delta_{1}^{(1)} \backslash \Delta_{1}^{(2)}\right)<\varepsilon_{1}$, где $\mu(X)$ - мера Лебега множества $X$, и далее множество

$$
\Delta_{2}^{(1)} \subset\left(\left(k_{\|}^{(2)}\right)^{2}, \infty\right) \backslash \mathcal{M}_{1}\left(k_{\|}^{(2)}\right),
$$

состоящее из конечного числа замкнутых промежутков, выбранных так, чтобы выполнялось включение $\Delta_{1}^{(1)} \subset \Delta_{1}^{(2)} \cup \Delta_{2}^{(1)}$. Для $k_{\|}=k_{\|}^{(1)}, E \in \Delta_{1}^{(2)}$, а также для $k_{\|}=k_{\|}^{(2)}$, $E \in \Delta_{2}^{(1)}$ сушествуют решения $\varphi(x)$ уравнения $(10)$ в случае $V(x)=V_{1}(x)$ и $N=1$ (см. начало доказательства). В $\Omega_{2}$ эти решения удовлетворяют уравнению (10) для $V(x)=V_{1}(x)$ и $N=2$; действительно, интегралы в правой части уравнений приводятся к одному и тому же интегралу по $\mathbb{R}^{3}$. Выберем теперь некоторую точку $k_{\|}^{(3)} \in \Omega_{2}^{*}$ с тем, чтобы $k_{1}^{(3)} \neq 0$ для всех образов, и множество $\Delta_{3}^{(1)}=\left[a_{2}, a_{1}\right] \cup\left[b_{1}, b_{2}\right]$, где

$$
a_{2} \in\left(\left(k_{\|}^{(3)}\right)^{2}, \min \left\{1 / 2, a_{1}\right\}\right), \quad b_{2}>\max \left\{b_{1}, 2\right\} .
$$

Заметим, что множество $\mathcal{M}_{1}\left(k_{\|}\right)$при рассмотрении оператора $H_{1}\left(k_{\|}\right)$в $L^{2}\left(\Omega_{2}\right)$ увеличивается. Как и выше, для $\varepsilon_{1}$ и (достаточно малых) $\varepsilon_{2}, \varepsilon_{3}>0$ строим множества $\Delta_{1}^{(3)}$, $\Delta_{2}^{(2)}, \Delta_{3}^{(2)}$, полученные соответственно из множеств $\Delta_{1}^{(2)}, \Delta_{2}^{(1)}, \Delta_{3}^{(1)}$ выбрасыванием интервалов, содержащих точки, соответственно $\mathcal{M}_{1}\left(k_{\|}^{(1)}\right), \mathcal{M}_{1}\left(k_{\|}^{(2)}\right), \mathcal{M}_{1}\left(k_{\|}^{(3)}\right)$ (здесь $\left.k_{\|}^{(j)} \in \Omega_{2}^{*}, j=1,2,3\right)$, столь малых, чтобы выполнялись неравенства

$$
\mu\left(\Delta_{1}^{(1)} \backslash \Delta_{1}^{(3)}\right)<\varepsilon_{1}, \quad \mu\left(\Delta_{2}^{(1)} \backslash \Delta_{2}^{(2)}\right)<\varepsilon_{2}, \quad \mu\left(\Delta_{3}^{(1)} \backslash \Delta_{3}^{(2)}\right)<\varepsilon_{3},
$$


а также одновременно с этим строим множества $\Delta_{4}^{(1)}, \Delta_{5}^{(1)}, \Delta_{6}^{(1)}$, состояшие из конечного числа замкнутых отрезков, не пересекаюшихся соответственно с множествами $\mathcal{M}_{1}\left(k_{\|}^{(4)}\right), \mathcal{M}_{1}\left(k_{\|}^{(5)}\right), \mathcal{M}_{1}\left(k_{\|}^{(6)}\right)$ для некоторых точек $k_{\|}^{(4)}, k_{\|}^{(5)}, k_{\|}^{(6)}$ из $\Omega_{2}^{*}$ с ненулевой первой координатой для всех образов и достаточно близких к точкам соответственно $k_{\|}^{(1)}, k_{\|}^{(2)}, k_{\|}^{(3)}$, и такие, что

$$
\Delta_{1}^{(2)} \subset \Delta_{1}^{(3)} \cup \Delta_{4}^{(1)}, \quad \Delta_{2}^{(1)} \subset \Delta_{2}^{(2)} \cup \Delta_{5}^{(1)}, \quad \Delta_{3}^{(1)} \subset \Delta_{3}^{(2)} \cup \Delta_{6}^{(1)} .
$$

Рассмотрим в $\Omega_{2}$ уравнение (10) для $N=2$ и $V(x)=V_{1}(x)$, а также $V(x)=V_{2}(x)$; соответствующие интегральные операторы в правой части (10), действующие на функции $\varphi \in L^{2}\left(\Omega_{2}\right)$, обозначим через $K_{1}$ и $K_{2}$. В силу леммы 6 для достаточно малых (в смысле этой леммы) $W_{2}(x)$ норма $\left\|K_{1}-K_{2}\right\|$ как угодно мала. По построению для точек $k_{\|}^{(i)}, i=1, \ldots, 6$, и для $E$ из соответствуюших множеств $\Delta_{1}^{(3)}, \Delta_{2}^{(2)}, \Delta_{3}^{(1)}, \Delta_{4}^{(1)}, \Delta_{5}^{(1)}$ и $\Delta_{6}^{(1)}$ существует ограниченный обратный оператор $\left(1-K_{1}\right)^{-1}$, а значит, существует $\left(1-K_{2}\right)^{-1}$, причем норма

$$
\left\|\left(1-K_{2}\right)^{-1}-\left(1-K_{1}\right)^{-1}\right\|
$$

сколь угодно мала в случае, если функция $W_{2}(x)$ достаточно мала. Следовательно, норма

$$
\left\|\varphi_{2}(x)-\varphi_{1}(x)\right\|_{L^{2}(\Omega)},
$$

где $\varphi_{1}(x), \varphi_{2}(x)$ - решения рассматриваемых уравнений, также сколь угодно мала. Для соответствующих функциям $\varphi_{1}(x), \varphi_{2}(x)$ решений $\psi_{1}(x), \psi_{2}(x)$ уравнений ЛиппманаШвингера норму $\left\|\psi_{1}(x)-\psi_{2}(x)\right\|_{L^{\infty}(\Omega)}$ также можно сделать сколь угодно малой; это получается с помощью простых оценок из вида данных уравнений, малости величины (15) и условия теоремы с использованием сформулированных в доказательстве леммы 6 утверждений.

Построение продолжается по индукции. На $(N+1)$-м шаге из полученных на $N$-м шаге для $k_{\|}^{(i)} \in \Omega_{N}^{*}$ сегментов выбрасываем интервалы, содержашие точки из $\mathcal{M}_{N}\left(k_{\|}^{(i)}\right)$, где $k_{\|}^{(i)} \in \Omega_{N+1}^{*}$, и добавляем новые сегменты, отвечающие одновременно добавляемым точкам $\bar{k}_{\|}^{(j)}$, закрывающие полученные шели и не содержащие точек из $\mathcal{M}_{N}\left(\bar{k}_{\|}^{(j)}\right)$. Затем решаем уравнение Липпмана-Швингера для данных $k_{\|}^{(i)}, \bar{k}_{\|}^{(j)}$ и $E$ из соответствуюших сегментов для $V(x)=V_{N}(x)$ и $V(x)=V_{N+1}(x)$ с тем, чтобы найденные решения отличались достаточно мало по норме $L^{\infty}\left(\Omega_{N+1}\right)$ (в том числе решения для $V(x)=V_{N+1}(x)$ от решений, найденных на предыдушем шаге). Далее добавляем новые сегменты вида $\left[a_{N+1}, a_{N}\right],\left[b_{N}, b_{N+1}\right]$ вместе с точкой $k_{\|}^{\prime} \in \Omega_{N+1}^{*}$, где

$$
a_{N+1} \in\left(\left(k_{\|}^{\prime}\right)^{2}, \min \left\{1 / N+1, a_{N}\right\}\right), \quad b_{N+1}>\max \left\{b_{N}, N+1\right\}
$$

при этом заботимся о том, чтобы мера объединения выброшенных на данном и всех предыдущих шагах интервалов из произвольного участвующего в построении и возникшего $M$-м по счету объединения сегментов, отвечающих одной точке, была меньше некоторого $\varepsilon=\varepsilon^{(M+1)} \rightarrow 0$ при $M \rightarrow \infty$. Пусть, далее, $N_{0}$ - шаг в построении, на котором впервые ищется решение $\psi_{N_{0}}(x)$ уравнения Липпмана-Швингера для точки $k_{\|}^{\left(N_{0}\right)} \in \Omega_{N_{0}}^{*}$ и 
соответствующего набора сегментов. Для данной точки $k_{\|}^{\left(N_{0}\right)}$ (и ее образов) и соответствуюших $E$ (множество таких $E$ от шага к шагу уменьшается) требуется выполнение неравенства

$$
\left\|\psi_{N_{0}}(x)\right\|_{L^{\infty}\left(\mathbb{R}^{3}\right)}>\sum_{N=N_{0}}^{\infty}\left\|\psi_{N+1}(x)-\psi_{N}(x)\right\|_{L^{\infty}\left(\mathbb{R}^{3}\right)},
$$

чего можно добиться (для всех таких точек одновременно) последовательным выбором достаточно малых $W_{N}(x), N>N_{0}$. Это обеспечивает нетривиальность решения

$$
\psi(x)=\psi_{N_{0}}(x)+\sum_{N=N_{0}}^{\infty}\left(\psi_{N+1}(x)-\psi_{N}(x)\right)
$$

“предельного" уравнения Шредингера (2), а также то, что $\psi(x)$ не стремится к нулю при $x_{3} \rightarrow \pm \infty$. Предельная периодичность функции $|\psi(x)|$ вытекает из периодичности функций $\psi_{N}(x)$. Из процедуры построения и условия $\lim _{M \rightarrow \infty} \varepsilon^{(M)}=0$ следует, что для любого появившегося на каком-либо шаге сегмента мера множества точек, принадлежащих ему, для которых имеется решение уравнения (2), равна длине отрезка. Объединение всех таких сегментов совпадает с $(0, \infty)$, и тем самым теорема доказана.

\section{Список литературы}

[1] Davies E. B. // Proc. Cambr. Philos. Soc. 1977. V. 82. P. 327.

[2] Чубурин Ю. П. О рассеянии на кристаллической пленке: Препринт. Свердловск: ФТИ УНЦ AH CCCP, 1985

[3] Рид М., Саймон Б. Методы современной математической физики. Т. 4. М.: Мир, 1982.

[4] Чубурин Ю. П. // ТМФ. 1987. Т. 72. №1. С. 120.

[5] Вольф Г. В., Чубурин Ю. П., Рубцова Л. А. // Поверхность. 1991. №10. С. 81.

[6] Чубурин Ю. П. // ТМФ. 1995. Т. 102. № 1. С. 74.

[7] Avron J., Simon B. // Commun. Math. Phys. 1981. V. 82. P. 101.

[8] Рид М., Саймон Б. Методы современной математической физики. Т. 1. М.: Мир, 1977.

[9] Козлов С. М. // Тр. Моск. матем. об-ва. 1983. Т. 46. С. 99.

[10] Simon B. // Bull. Amer. Math. Soc. 1982. V. 7. P. 447.

[11] Чубурин Ю. П. // Матем. заметки. 1992. Т. 52. Вып. 2. С. 138.

[12] Рид М., Саймон Б. Методы современной математической физики. Т. 3. М.: Мир, 1982.

[13] Чубурин Ю. П. // ТМФ. 1994. Т. 98. №1. С. 38.

Физико-технический институт

Удмуртского научного центра

Поступила в редакцию

Уральского отделения

Российской академии наук

21.III.1995 г.

\section{Yu.P. Chuburin \\ ON SCHRODINGER EQUATION FOR THE PLANE FILM WITH THE LIMIT PERIODIC LATTICE}

The solutions of the Schrodinger equation with the potential that is limit periodic in $x_{1}, x_{2}$ and that decreases when $\left|x_{3}\right| \rightarrow \infty$ are constructed and some of their properties are described. 